\title{
Malignant Mixed Mullerian Tumor of Fallopian Tube: A Case Report
}

\begin{abstract}
We report a case of malignant mixed mullerian tumor (MMMT) of fallopian tube in a 49 year old female who presented to our institute in November 2014 with the chief complaints of irregular and excessive bleeding per vaginum for 3 months. MRI of the patient revealed a right adenexal complex mass of 9 x 5 x $7 \mathrm{~cm}$. Her CA125 level was $136 \mathrm{U} / \mathrm{ml}$, whileCA19-9 and CEA levels were normal. Her cervical cytology was normal and preoperative endometrial curetting revealed secretory endometrium. A malignant ovarian tumor was suspected and the patient was taken up for staging surgery. On final histopathology it was MMMT of right fallopian tube, stage IA. She received 6 cycles of cisplatin, ifosfamide and paclitaxel (TIP) based chemotherapy. Presently she is alive with no evidence of disease with a follow up period of 28 months. This case report suggests that prognosis of early stage MMMT is good, especially if there is no deep infiltration of the muscle layer of fallopian tube and the peritoneal cytology is negative. Chemotherapy using a platinum drug after surgery seems to be the most effective treatment in this disease.
\end{abstract}

Keywords: MMMT of fallopian tube, Platinum based chemotherapy, Staging surgery
Volume 7 Issue $6-2017$

\author{
Vandana Jain, Rupinder Sekhon, Shveta Giri, \\ Sudhir Rawal \\ Department of Uro-Gynae Oncology, Rajiv Gandhi Cancer \\ Institute and Research Centre, India
}

\begin{abstract}
Correspondence: Vandana Jain, Fellow Gynecologic Oncology, Rajiv Gandhi Cancer Institute and Research Centre, Sector 5, Rohini, New Delhi, India, Tel 919910700788 , Fax 91-II-
\end{abstract} 2705I037,Email dr.vandana.j@gmail.com

Received: April 18, 2017| Published: August 15, 2017
Abbreviations: B/L, Bilateral; TAH, Total Abdominal Hysterectomy; BSO, Bilateral salpingo-oopherectomy

\section{Introduction}

Malignant tumors of fallopian tube are very rare. The most common histological type is adenocarcinoma. Sarcomas occur infrequently and carcinosarcomas or Malignant mixed mullerian tumors (MMMT) are very rare. These highly aggressive tumors are composed of both malignant epithelial and stromal elements and usually have poor prognosis. Most patients of MMMT fallopian tube present with nonspecific symptoms like abdominal pain or distension or abnormal vaginal bleeding. In a great majority of cases the diagnosis is not made until the time of surgery or histological evaluation. Data are limited, but primary cytoreductive surgery followed by platinum based chemotherapy seems to be effective.

\section{Case presentation}

We present the case of 49 year old perimenopausal female, P2L2, who came to our institute in November 2014 with the chief complaints of irregular and heavy bleeding for the past 3 months. On examination the general condition of patient was stable with a pulse rate of $88 /$ minute and blood pressure of $128 / 78 \mathrm{~mm} \mathrm{Hg}$. On per-abdomen examination, a mass was palpable in the lower abdomen arising from the pelvis and the same mass was palpable on the pervaginal examination. Her CA125 level was $136 \mathrm{U} / \mathrm{ml}$ while her CA199 and CEA levels were normal. Her CBC was normal, Hb-9.9 g/dl, TLC-9000/mm3, Platelet count-2.3 lakh/mm3. MRI suggested a right adenexal mass of $9.3 \times 5.4 \times 6.6 \mathrm{~cm}$ adherent to the adjacent rectal wall with no other evidence of disease. Endometrial biopsy revealed secretory endometrium with no evidence of malignancy. Cervical cytology was also normal. A pre-operative diagnosis of malignant ovarian tumor was made.

She underwent exploratory laparotomy. Per-operatively the upper abdomen was normal. About $50 \mathrm{ml}$ of haemorrhagic fluid was present in the pelvis. Left fallopian tube was dilated to about
$10 \times 5 \mathrm{~cm}$ with smooth outer surface. Bilateral ovaries, uterus and right tube was apparently normal while the bladder peritoneum appeared suspicious. She underwent total abdominal hysterectomy with bilateral salpingo-oopherectomy (TAH with BSO), which was malignant on frozen section and this was followed by $\mathrm{B} / \mathrm{L}$ pelvic nodal dissection, retroperitoneal lymph node sampling, total omentectomy, appendicectomy, bladder peritonectomy, multiple peritoneal biopsies and fluid for cytology.

\section{Histopathological findings}

Gross description- Right fallopian tube was enlarged and expanded to $9 \times 5 \times 5 \mathrm{~cm}$. The serosal surface was smooth with no breach. The luminal surface showed a friable, polypoidal, yellowish white haemorrhagic tumor $8.3 \times 4.8 \times 4.7 \mathrm{~cm}$.

Histological description: The tumor was composed of malignant epithelial as well as mesenchymal component. The epithelial component was in the form of high grade endometrioid adenocarcinoma. The mesenchymal component was composed of high grade spindle cells with marked pleomorphism, brisk mitosis and extensive areas of necrosis (Figure 1). The tumor was infiltrating into the wall of fallopian tube, however serosal breach was not identified. Lymphovascular invasion was not seen. B/L ovaries, left fallopian tube, uterus, omentum, appendix, peritoneal biopsies, bladder peritoneum, pelvic and retroperitoneal lymph nodes were free of tumor. Peritoneal fluid was negative for malignant cells. So a final diagnosis of MMMT (carcinosarcomas) of fallopian tube, stage IA (FIGO 2014) was made. The patient received 6 cycles of cisplatin, ifosfamide and paclitaxel (TIP) based chemotherapy and presently the patient is alive with no evidence of disease with a follow up period of about 28 months.

\section{Discussion}

Primary MMMT of fallopian tube are rare (less than $4 \%$ of all gynaecological carcinosarcomas), which most frequently occur in the endometrium, vagina, cervix and ovary in descending order of 
frequency. ${ }^{1,2}$ In our study the age of the patient was 49 yrs. In a review most patients were in the fifth or sixth decade of life, with a mean age of 57.5 years. ${ }^{1}$ Most of the patients with MMMT of fallopian tube present with nonspecific symptoms. In a review, frequent symptoms were abdominal pain or distension (47.9\%) followed by atypical genital bleeding $(34.2 \%){ }^{3}$ It has been noted that postmenopausal bleeding or spotting with negative cervical cytology and endometrial curetting should raise the suspicion of tubal malignancy, ${ }^{4}$ as was the case in our patient. The diagnosis is usually not made until the time of surgery, with the most common pre-operative diagnosis being ovarian malignancy. ${ }^{1}$ Our patient was also diagnosed as malignant ovarian tumor prior to surgery.

Surgical procedures at laparotomy should include collection of washings for cytology, a thorough exploration of all peritoneal surfaces, omentectomy, pelvic and retroperitoneal lymph node sampling and peritoneal biopsies in addition to TAH with BSO. A maximal effort at tumor cytoreduction is emphasized similar to ovarian cancer and adenocarcinoma of fallopian tube. ${ }^{5}$

The prognosis of patients with MMMT of fallopian tube is usually poor. Extra tubal spread is the most important prognostic factor which is same as primary adenocarcinoma of fallopian tube. MMMT of fallopian tube is usually diagnosed at an advanced stage. In a recent review only about $32.3 \%$ patients were stage I. The 3 year survival was $62.7 \%$ for stage I/II patients and $39.8 \%$ for stage III/IV patients. ${ }^{3}$ There is increasing evidence that adjuvant platinum based chemotherapy used alone or in combination with radiotherapymay improve the survival in cases of tubal MMMT., ${ }^{2,3,6}$ In a recent review, Ifosfamide based chemotherapy (IEP) appeared to be a promising regiment for this disease. ${ }^{3}$ GOG has also reported a high level of benefit for ifosfamide and cisplatin in uterine carcinosarcomas. ${ }^{7}$ In the present case report the patient was stage I MMMT of fallopian tube and received platinum based chemotherapy (TIP) after staging surgery. The patient is alive with no evidence of disease after 28 months of follow up.

\section{Conclusion}

In conclusion, MMMT of fallopian tube is associated with poor prognosis, although in early stage the prognosis is not that bad, especially if there is no deep infiltration of the wall of fallopian tube. Primary cytoreductive surgery followed by adjuvant platinum based chemotherapy is the most effective treatment available at present.

\section{Acknowledgements}

We would like to say special thanks to Dr. Anila Sharma and Dr. Parul Tanwar for providing the histopathology pictures to us.

\section{Conflicts of Interest}

No financial interest or conflicts of interest exist.

\section{References}

1. Imachi M, Tsukamoto N, Shigematsu T, et al. Malignant mixed mullerian tumor of the fallopian tube: report of two cases and review of literature. Gynecol Oncol. 1992;47(1):114-124.

2. Horn LC, Werschnik C, Bilek $\mathrm{K}$, et al. Diagnosis and clinical management in malignant mixed mullerian tumors of the fallopian tube. Arch Gynaecol Obstet. 1996;258(1):47-53.

3. Yokoyama Y, Yokota M, Futagami M, et al. Carcinosarcoma of the fallopian tube: Report of four cases and review of literature. Asia Pac J Clin Oncol. 2012;8(3):303-311.

4. Kinoshita M, Asano S, Yamashita M, et al. Mesodermal mixed tumor primary in the fallopian tube. Gynecol Oncol. 1989;32(3):331-335.

5. Shen YM, Xie YP, Xu L, et al. Malignant mixed mullerian tumor of the fallopian tube: report of two cases and review of literature. Arch Gynecol Obstet. 2010;281(6):1023-1028.

6. Weber AM, Hewett WF, Gajewski WH, et al. Malignant mixed mullerian tumors of the fallopian tube. Gynecol Oncol. 1993;50(2):239-243.

7. Sutton G, Brunetto VL, Kilgore L, et al. A phase III trial of ifosfamide with or without cisplatin in carcinosarcoma of the uterus: a Gynecologic Oncology Group Study. Gynecol Oncol. 2000;79(2):147-153. 